\title{
A novel method for detection of pigment network in dermoscopic images using graphs
}

\author{
Maryam Sadeghi ${ }^{a, b, *}$, Majid Razmara ${ }^{a}$, Tim K. Lee ${ }^{a, b, c}$, M.Stella Atkins ${ }^{a}$ \\ a Simon Fraser University, 8888 University Drive, Burnaby, BC, Canada V5A1S6 \\ ${ }^{\mathrm{b}}$ Cancer Control Research, BC Cancer Agency, 675 W. 10th Ave., Vancouver, Canada \\ ${ }^{\mathrm{c}}$ Department of Dermatology and Skin Science, and Photomedicine Institute, University of British Columbia, and Vancouver Coastal Health Research Institute, Canada
}

\section{A R T I C L E I N F O}

\section{Article history:}

Received 12 November 2009

Received in revised form 3 March 2010

Accepted 13 July 2010

\section{Keywords:}

Dermoscopic structures

Pigment network detection

Texture analysis

Dermoscopy

Melanoma

Computer-aided diagnosis

Graph

\begin{abstract}
A B S T R A C T
We describe a novel approach to detect and visualize pigment network structures in dermoscopic images, based on the fact that the edges of pigment network structures form cyclic graphs which can be automatically detected and analyzed. First we perform a pre-processing step of image enhancement and edge detection. The resulting binary edge image is converted to a graph and the defined feature patterns are extracted by finding cyclic subgraphs corresponding to skin texture structures. We filtered these cyclic subgraphs to remove other round structures such as globules, dots, and oil bubbles, based on their size and color. Another high-level graph is created from each correctly extracted subgraph, with a node corresponding to a hole in the pigment network. Nodes are connected by edges according to their distances. Finally the image is classified according to the density ratio of the graph. Our results over a set of 500 images from a well known atlas of dermoscopy show an accuracy of $94.3 \%$ on classification of the images as pigment network Present or Absent.
\end{abstract}

Crown Copyright @ 2010 Published by Elsevier Ltd. All rights reserved.

\section{Introduction}

Melanoma, a cancerous lesion in the pigment-bearing basal layers of the epidermis, is the most deadly form of skin cancer, yet it is also the most treatable, with a cure rate for early-stage melanoma of almost $100 \%$. Therefore, there is a need to develop computer-aided diagnostic systems to facilitate the early detection of melanoma. The first step in these systems is skin lesion segmentation which is widely addressed in the literature $[1,2]$. The next essential step is feature extraction and pattern analysis procedures to make a diagnosis. According to the literature, pigment network or reticular pattern is an important diagnostic parameter for melanoma [3]. In this paper, we address the problem of how to determine the absence or presence of pigment networks in a given dermoscopic image. A typical pigment network is defined as "a light-to-darkbrown network with small, uniformly spaced network holes and thin network lines distributed more or less regularly throughout the lesion and usually thinning out at the periphery" [3].

Our proposed method is a robust, reliable, computer-aided diagnostic tool for analyzing the texture in lesions of the skin to detect pigment networks in the presence of other structures such as dots

\footnotetext{
* Corresponding author at: Computing Science, Simon Fraser University, 8888 University Drive, Burnaby, BC, Canada V5A1S6. Tel.: +1 7787825509.

E-mail address: maryam_sadeghi@sfu.ca (M. Sadeghi).
}

and globules. The goal is to classify a given image to one of two classes: Present or Absent, that has qualitative information about the absence or presence of the pigment network. Fig. 1a and $c^{1}$ show examples of Present and Absent lesions and 1b and 1d show both images enlarged.

Detection of the pigment network has been investigated recently [4-8]. Fleming et al. [4] report the detailed techniques for extracting and measuring the pigment network characteristics. They investigated the thickness and the variability of thickness of network lines; the size and variability of network holes; and the presence or absence of radial streaming and pseudopods near the network periphery. They use morphological techniques in their method and their results are purely qualitative.

Anantha et al. [7] propose two algorithms for detecting pigment networks in skin lesions: one involving statistics over neighboring gray-level dependence matrices and one involving filtering with Laws energy masks. Various Laws masks are applied and the responses are squared. Improved results are obtained by a weighted average of two Laws masks whose weights are determined empirically. Classification of these tiles is done with approximately $80 \%$ accuracy.

Betta et al. begin by taking the difference of an image and its response to a median filter [8]. This difference image is thresholded

\footnotetext{
${ }^{1}$ For higher resolution, colorful images refer to the online version.
} 
(a)

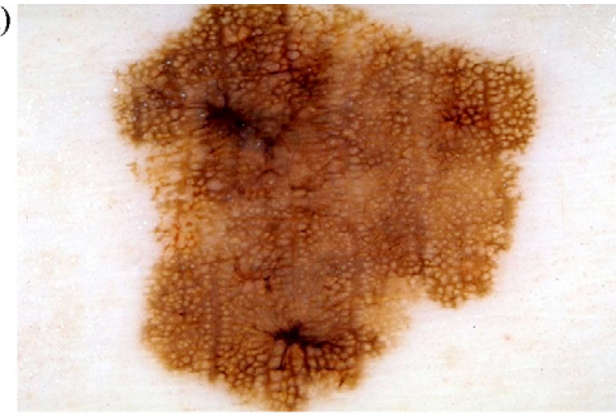

(c)

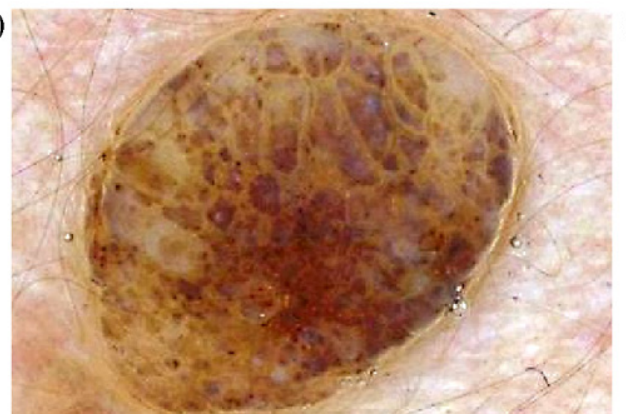

(b)

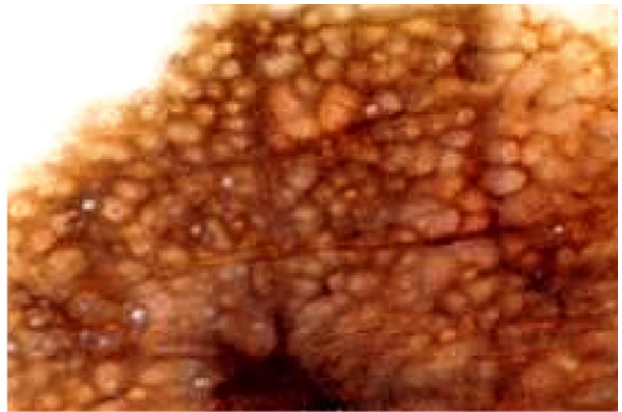

(d)

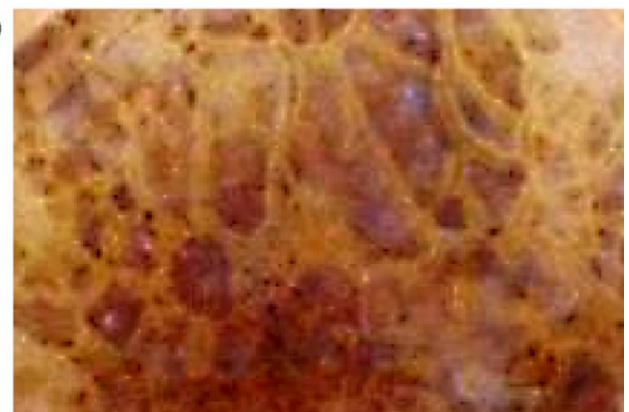

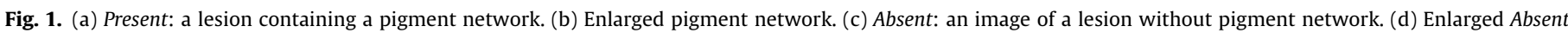
image.

to create a binary mask which undergoes a morphological closing operation to remove any local discontinuities. This mask is then combined with a mask created from a high-pass filter applied in the Fourier domain to exclude any slowly modulating frequencies. Results are reported graphically, but appear to achieve a sensitivity of $50 \%$ with a specificity of $100 \%$.

\section{Methods overview}

We analyze a set of lesion images each with a label (Present or Absent) representing the presence of a pigment network in the image. The specific location of the pigment network structure within the image is found and visualized. Then, the image is classified according to the ratio of the pigment network presence.

Fig. 2 shows an overview of our novel graph-based approach in which after a pre-processing step, sharp changes of intensity are detected using the Laplacian of Gaussian (LOG) filter. The result of this edge detection step is a binary image which is subsequently converted into a graph to find meshes or cyclic structures of the lesion. After finding loops or cyclic subgraphs of the graph, noise or undesired cycles are removed and a graph of the pigment network is created using the extracted cyclic structures. According to the density of the pigment network graph, the given image is classified into Present or Absent.

\subsection{Pre-processing}

First, in order to prevent unnecessary analysis of the pixels belonging to the skin, the lesion is manually segmented. Concurrently we developed an automatic segmentation method for dermoscopic images [2], but it was not ready for our experiment, although we would use it in future work. Next, the quality of the image is enhanced to highlight texture features. For sharpening, we use the MATLAB Image Processing Tool Box function Unsharp mask. Unsharp mask is one of the most popular tools for image sharpening [9]. A two-dimensional high-pass filter is created using Eq. (1). This high-pass filter sharpens the image by removing the low frequency noise. We used the default parameter of the MATLAB in our experiment. Fig. 3b shows the result of the sharpening step.

Sharpening filter $(\alpha)=\left(\frac{1}{\alpha+1}\right)\left|\begin{array}{ccc}-\alpha & \alpha-1 & -\alpha \\ \alpha-1 & \alpha+5 & \alpha-1 \\ -\alpha & \alpha-1 & -\alpha\end{array}\right|$.

To investigate structures of the skin texture, it was necessary to reduce the color images to a single plane before applying our algorithm. Various color transforms (NTSC, $L^{*} a^{*} b$, red, green, and blue channels separately, gray (intensity image), etc.) were investigated for this purpose. After the training and validation step, we selected the green channel as the luminance image. Results of the different color transformations are reported in Section 3.

\subsection{Feature extraction}

The presence of atypical pigment network is indicated by black, brown, or gray network with irregular meshes and thick lines and a typical pigment network is defined as a light-to-dark-brown network with small, uniformly spaced network holes and thin network lines distributed more or less regularly throughout the lesion and usually thinning out at the periphery.

These structures show prominent lines, homogeneous or inhomogeneous meshes. From an image processing point of view, these structures can be detected by searching for their occurrence and then validating their color and spatial characteristics. To extract these key features we search for round or cyclic structures which represent the presence of the pigment network. To find the meshes or cyclic structures in the single plane image, it is necessary to detect sharp changes of the intensity. For this purpose, we used the Laplacian of Gaussian filter. Because of the inherent properties of the Laplacian of Gaussian (LOG) filter, it can detect the "lightdark-light" changes of the intensity well. The detection criterion is the presence of a zero crossing in the second derivative with the corresponding large peak in the first derivative. We follow the MATLAB implementation of the LOG edge detection which looks for zero crossings and their transposes. All zeros are kept and edges lie on the zero points. If there is no zero, it arbitrarily chooses the edge to be the negative point. Therefore when all zero responses of the filtered image are selected, the output image includes all 


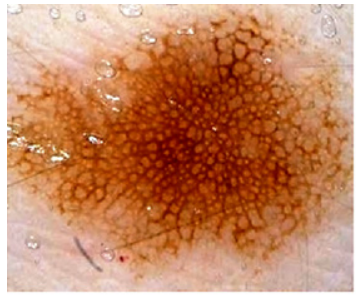

Original

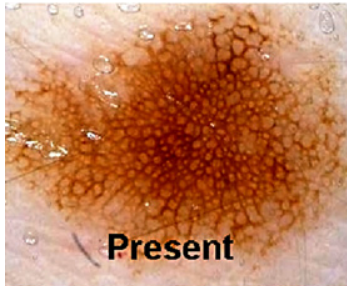

Classification

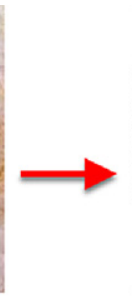

Laplacian of Gaussian
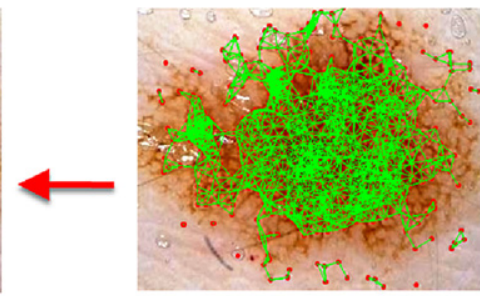

Pigment Network
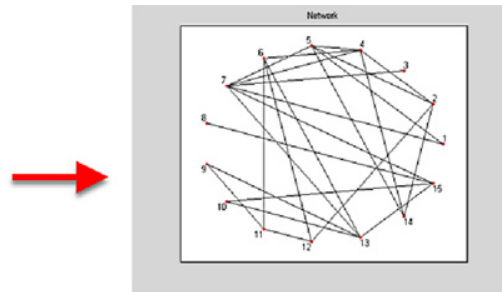

Image to Graph
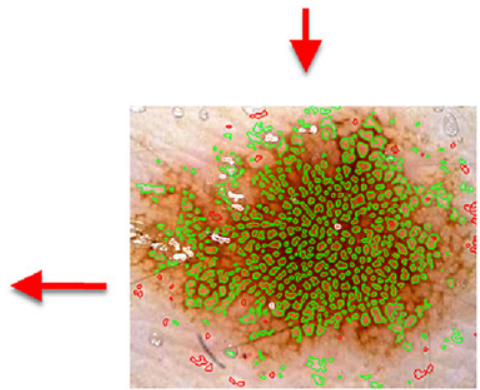

Cyclic Subgraphs

Fig. 2. Overview of the proposed algorithm.

closed contours of the zero crossing locations. An example of the edge detection step is shown in Fig. 3. This black and white image captures the potential meshes of the pigment networks. The LOG filtering step is restricted to the area within the lesion boundary, which was pre-determined. As a result, the LOG operation generates a closed contour surrounding the segmented lesion in Fig. 3c.

Now, meshes or round shaped structures in the image of edges are the goal features to be extracted. In previous work $[4,8,10]$ these structures usually are found by morphological techniques and a sequence of closing and opening functions applied to the black and white image. We did not use this approach because using morphological techniques is error-prone in detecting the round shape structures. So, having a binary image of the connected components (the edges of the image), each of them is converted to a graph $\left(G_{i}\right)$ using 8-connected neighboring. Each pixel in the connected component is a node of $G_{i}$ and each node has a unique label according to its coordinate.

To find the cyclic texture features, all cyclic subgraphs are detected using the Iterative Loop Counting Algorithm (ILCA) [11]. This algorithm transforms the network into a tree and does a depth first search on the tree for loops.

After finding cyclic subgraphs which represent meshes of the skin texture that could possibly belong to a pigment network, these subgraphs were filtered and noise or wrongly detected structures (globules and dots) were removed according to parameters learned in a training and validation step. We used two threshold parameters: one for the size and the other for the mean intensity difference between the inside and the border intensity of the meshes extracted from the edge image. Therefore, by setting a threshold for the difference between the average intensity of inner pixels and the average intensity of the border, we can discriminate globules from holes of the pigment network. First, we remove all detected cycles which are shorter than 7 pixels and longer than 150 pixels. These parameters can be set for a given data set according to the scale, magnification and resolution of images. The image set [12] does not provide precise information about the resolution and magnification of the image set which is used in our experiment. Furthermore, we are uncertain if the resolution and magnification is the same for all images of the atlas. Therefore, to play safe, we set a wide range (7-150) to find as many meshes of pigment networks as possible. For belonging to a pigment network, a detected cyclic subgraph should have a higher intensity on the area contained by the network structures (holes) than on the network itself (lines), but in globules and brown dots, the mean intensity of the area inside the structure is lower than the intensity of border pixels so we can discriminate them. We also have to deal with oil bubbles and white cysts and dots. These structures are similar to holes of the pigment network in terms of the mean intensity of the inside and border area, but they have much brighter inside. So, if there is at least one pixel with high intensity (set to 0.8 , in a scale ranging from 0 to 1 ) in the inside area of a mesh, it will be colored as white representing oil bubbles, white cysts or dots. Therefore, these wrongly detected round structures of brown dots and globules, white dots, white cysts and oil bubbles are removed from the rest of the analysis. We colored these noise structures as red and white meshes in Fig. 4. Using the training step we set these thresholds to 0.1 and (a)

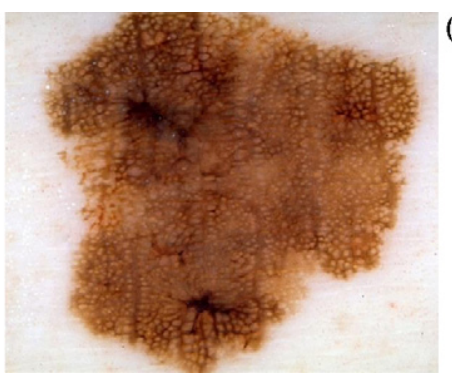

(b)

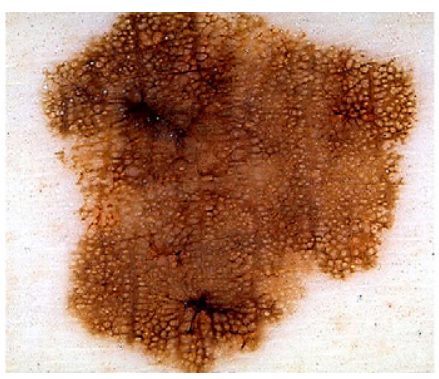

(c)

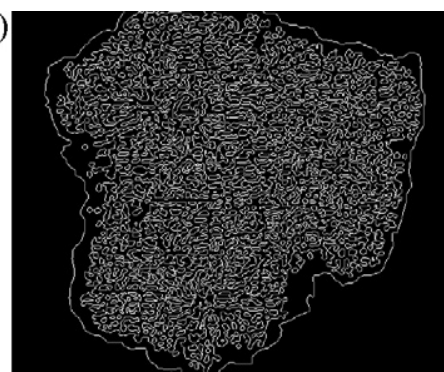

Fig. 3. (a) A given skin lesion image. (b) Sharpened image. (c) Result of the edge detection after segmenting the lesion. 
(a)

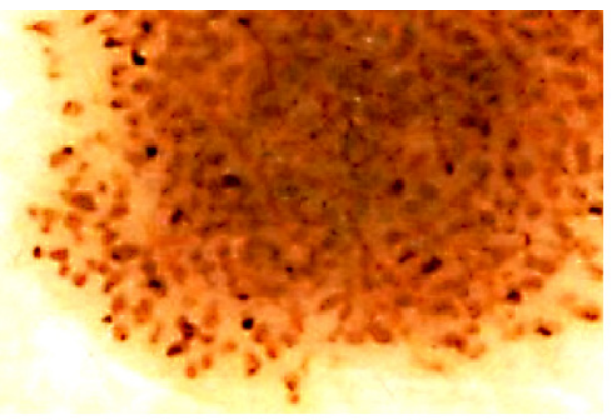

(c)

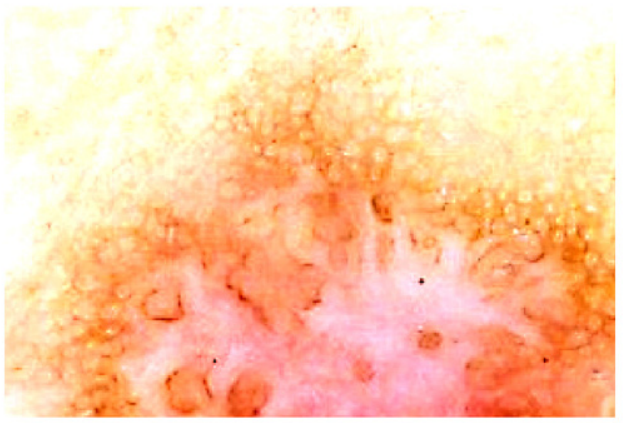

(e)

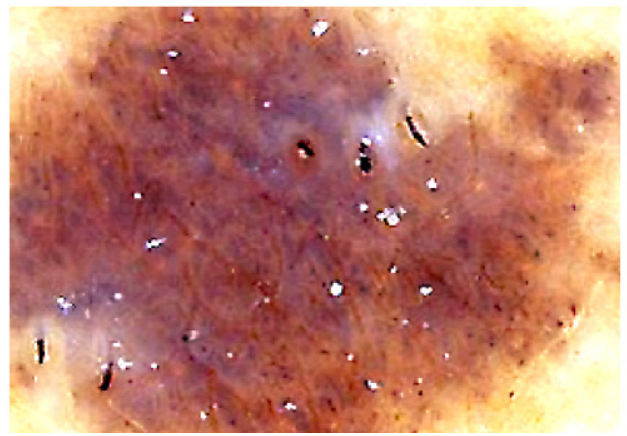

(b)

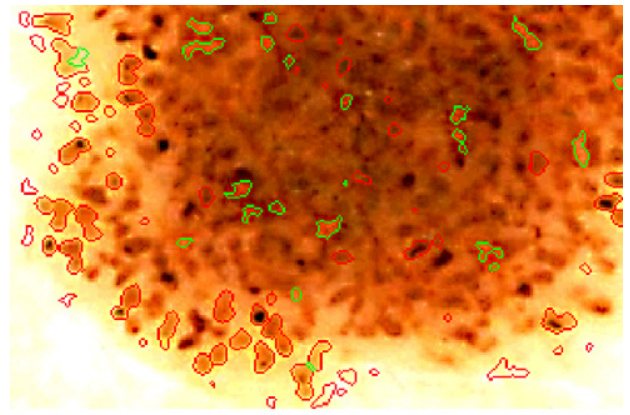

(d)

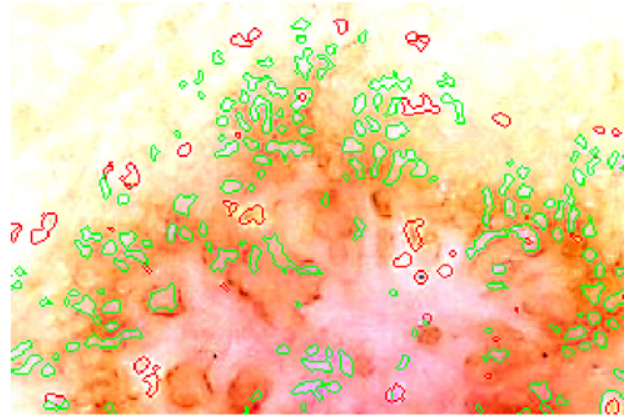

(f)

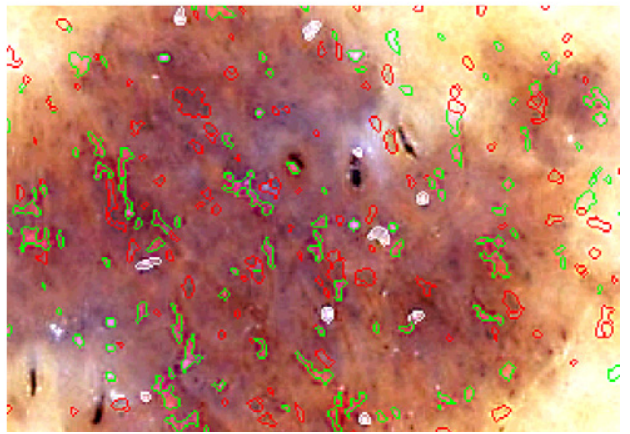

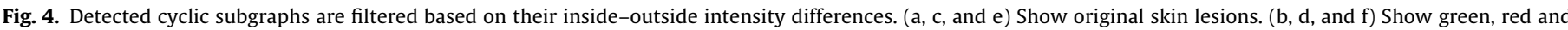

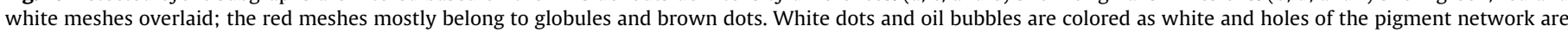
visualized as green.

0.01 for white and green meshes respectively which means:

Color $= \begin{cases}\text { Green } & 0.01<(I-B)<0.1 \\ \text { White } & 0.1<(I-B) \wedge \exists P_{I}>0.8 \\ \text { Red } & \text { Otherwise }\end{cases}$

where $I$ is mean intensity of the inside area, $B$ is the mean intensity of the border or outside area, and $P_{I}$ is a pixel inside the mesh. Fig. 4 shows three examples of skin lesions with filtered cyclic subgraphs overlaid. These structures can be used later for the analysis of globules and dots which are other important structures of the skin lesion texture.

In order to visualize the graph of a pigment network, we created a new higher-level graph whose nodes are centers of the meshes belonging to the pigment network (green meshes). Nodes within a maximum distance threshold (MDT) are connected together. However, there is not a minimum node distance threshold. The value of the MDT is computed based on the average diameter of all meshes in the image. Based on the pigment network definition, meshes of the network are uniformly spaced. To consider this spatial arrangement, the MDT should be proportional to the size of holes and it is defined as alpha (set to 3 ) times the average diameter of meshes.
To classify images into Absent and Present, we defined a density ratio as

Density $=\frac{|E|}{|V| \times \log (\text { LesionSize })}$

where $E$ is the number of edges in the graph, $V$ is the number of nodes of the graph and LesionSize is the size of the area of the image within the lesion boundary, being investigated for finding the pigment network. Being present is proportional to the number of edges since a bigger $|E|$ means that more holes are closer that MDT. Also, having a smaller $|V|$ for a fixed $|E|$ means that nodes or holes are uniformly spaced close to each other and graph of the pigment network is dense. Therefore, based on the pigment network definition, having a high density ratio is a requirement for being Present. $L$ esionsize is used to normalize the ratio $|E| /|V|$. For example, a fixed number of vertices and edges in a small lesion is more likely representing Present than in a relatively big lesion. However, since there is not a linear relationship between the size of a lesion and the probability of being Present or Absent, we found experimentally that the logarithm of LesionSize is more appropriate. Images containing a density ratio higher than a threshold (set to 1.5) are classified as Present and the rest as $A$ bsent. 
(a)

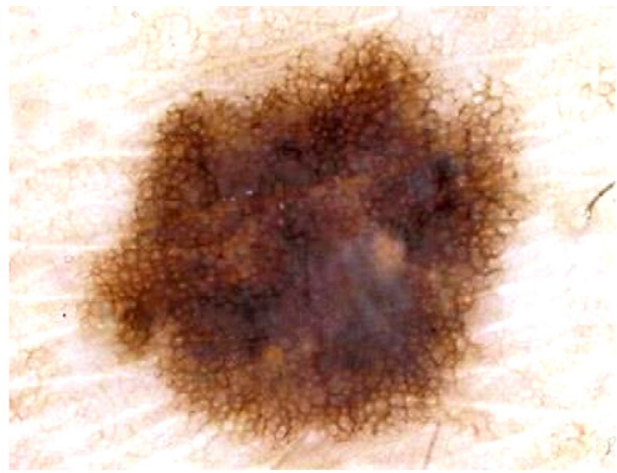

Original Image

(c)

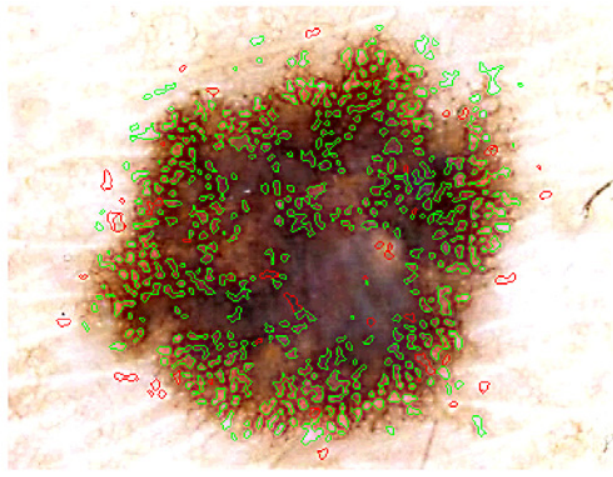

Cyclic Subgraphs

(e)

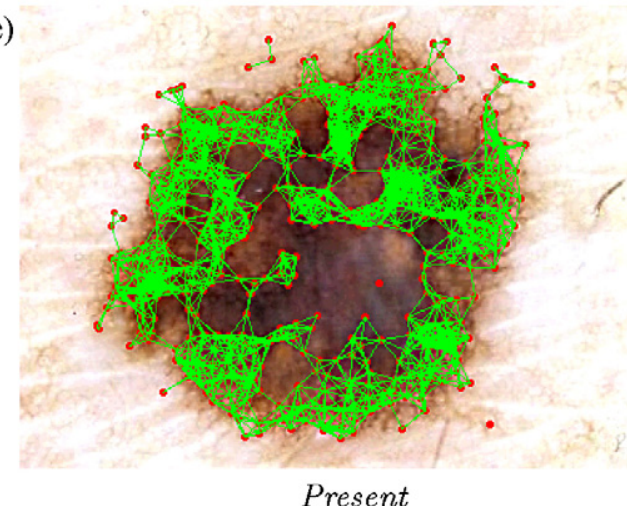

(b)

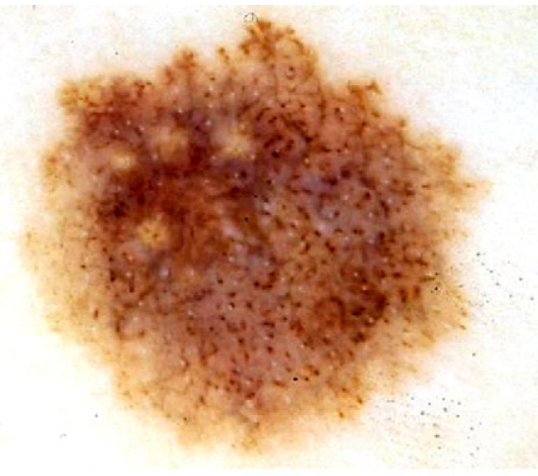

Original Image

(d)

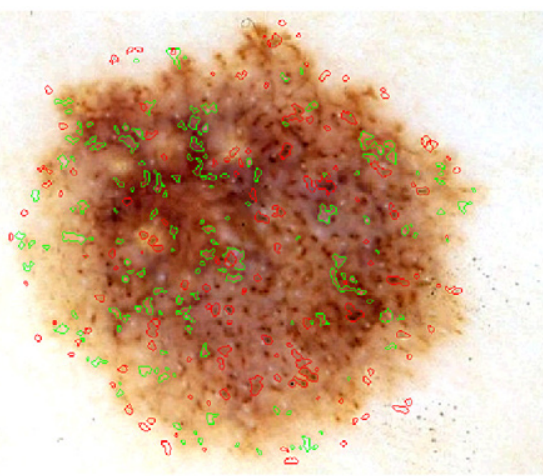

Cyclic Subgraphs

(f)

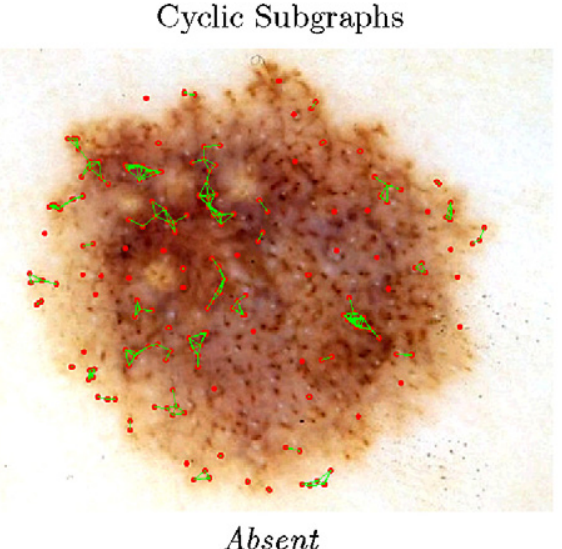

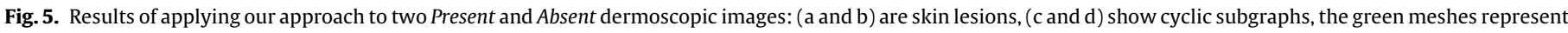

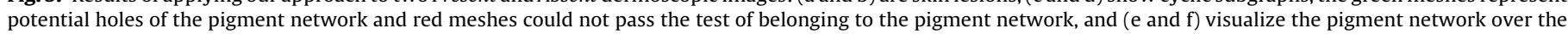
image.

\section{Evaluation and results}

We applied our method to a set of dermoscopic images taken from Argenziano et al.'s Interactive Atlas of Dermoscopy [12]. We tuned the parameters and thresholds of our proposed method according to a set of 100 images of size $768 \times 512$. Then we tested the method for another set of images (500 images) randomly selected from the atlas. Some of these images were challenging due to acquisition parameters such as lighting and magnification, being partial (entire lesion was not visible), or due to the presence of an unreasonable amount of occlusion by either oil or hair. These challenging images are usually discarded from test sets in the previous work. However, these images were kept in our test set. Fig. 5 illustrates two examples of our experimental results. The first column shows a Present image and the second one shows an Absent image, both of which are classified correctly. Table 1 shows the percentage of correct classifications (Present or $A$ bsent) for the 500 test images, using different color transformations. It is seen that the green channel gives the best classification. Comparing our results to Anantha et al.'s method, we achieved a better result, however the same gold standard is not used and the image sets are different. Therefore, a direct comparison is impossible due to different images and ground truths. Note that we deliberately created a difficult data set by not excluding oily, hairy and low-contrast images. Fig. 6 shows three difficult examples of our image set. Our method also locates the pigment network and provides a qualitative analysis which can be used for extraction of pigment network characteristics to discriminate typical pigment networks from atypical ones.

Table 1

Correct classification rates of various image transformations.

\begin{tabular}{lllllll}
\hline & $\mathrm{R}$ & $\mathrm{G}$ & $\mathrm{B}$ & $\mathrm{YIQ}$ & Gray & $L^{*} a^{*} b$ \\
\hline Correct classification & 90.7 & 94.3 & 90.1 & 92.6 & 91.1 & 89.7 \\
\hline
\end{tabular}


(a)

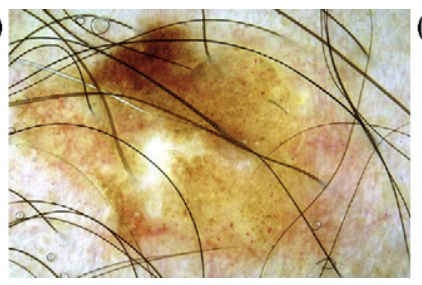

(b)

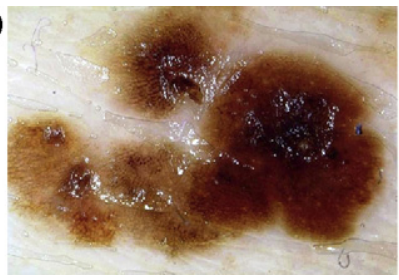

(c)

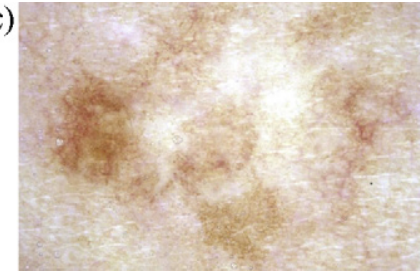

Fig. 6. Three difficult examples of our image set: (a) A hairy image. (b) An oily image. (c) A low-contrast image.

(a)

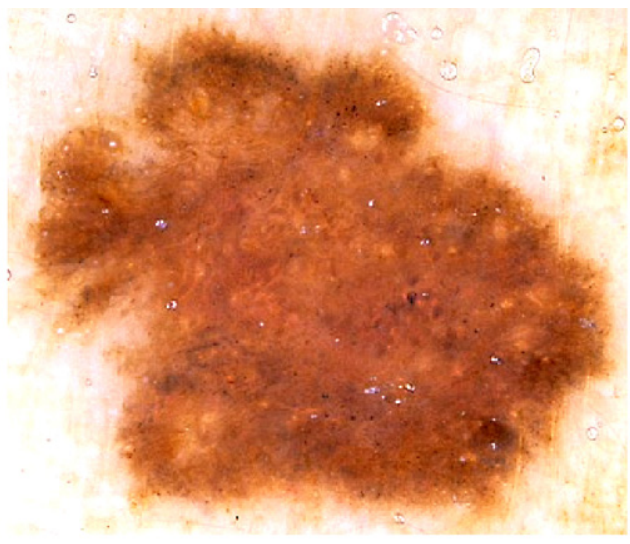

(c)

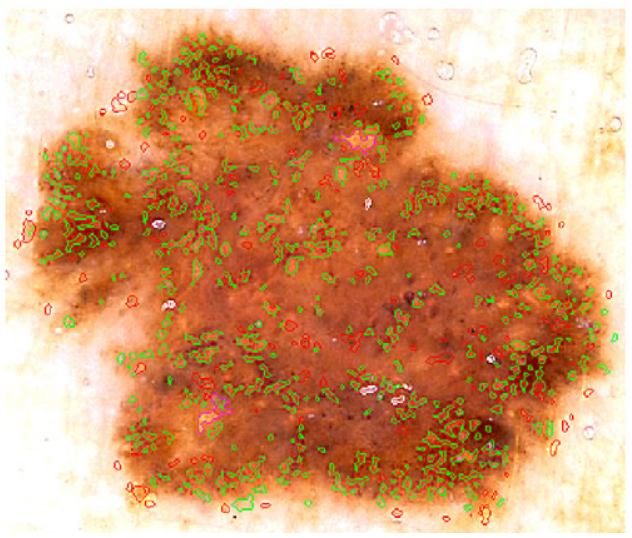

(b)

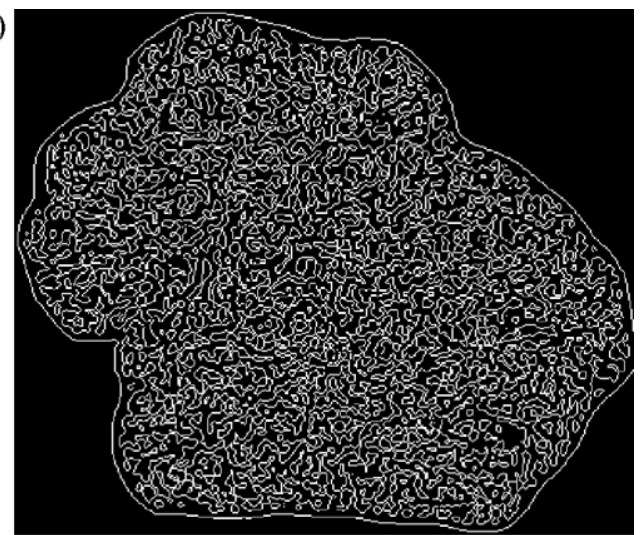

(d)

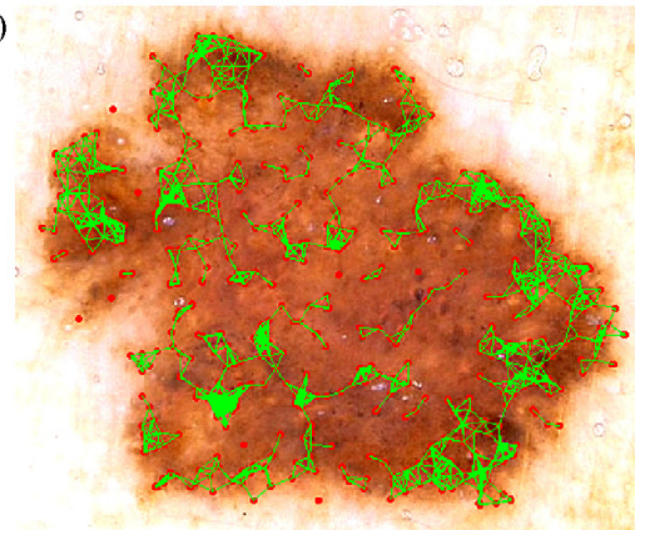

Fig. 7. A challenging image with $51.5 \%$ inter-expert agreement favoring the Present diagnosis.

According to the results of the various color transformations, the green channel is the best one for the pigment network detection. Interestingly YIQ (the transformation used for NTSC systems) has the second best result. The luminance $Y$ of the YIQ or NTSC transformation is defined as:

$Y=0.299 R+0.587 G+0.114 B$

where $R, G, B$ are the red, green, and blue color components, respectively. To compute the luminance $Y$, the green channel has larger weight than the other channels so is the likely reason the YIQ works well. In the gray-scale experiment, the intensity image is calculated by $(R+G+B) / 3$ and in the $L^{*} a^{*} b$ experiment, the L component of the $L^{*} a^{*} b$ space is used as the intensity image.

In some images, it is not easy to detect the pigment network even by experts. Fig. 7a shows one of these challenging images taken from [3], where the expert dermatologists only had 51.5\% agreement among themselves, with a small majority favoring the Present diagnosis. Our algorithm can assist dermatologists to make their diagnosis by visualizing the pigment network (if any). This method may also be used for training purposes. Fig. 7 illustrates the outcome of applying our method on the image, which classifies it as a Present pigment network.

\section{Conclusion and future work}

We proposed a novel graph-based method for classifying and visualizing pigment networks and validated the method by evaluating its ability to classify and visualize the real dermoscopic images. The accuracy of the system is $94.3 \%$ in classifying images to one of two classes of Absent and Present. This method can be used as a part of an automatic diagnosis system for classifying moles and detecting skin cancer. In addition, extracted features can be used in skin lesion segmentation systems. This is a novel idea that needs more investigation and evaluation and has a good potential for future research. Future work will include the separation of "typical" and "atypical" pigment networks, through analysis of the shape, size, color and thickness of the network lines. Furthermore, we believe that the same idea with different features can also be applied for extracting other skin patterns.

\section{Conflict of interest statement}

There is no conflict of interest. The funding agencies had no role in the manuscript. 


\section{Acknowledgments}

This work was funded by the Canadian Natural Sciences and Engineering Council (NSERC), CIHR-Skin Research Training Centre (SRTC) and a grant from the Canadian Health Research Project (CHRP).

\section{References}

[1] Celebi ME, Iyatomi H, Schaefer G, Stoecker WV. Lesion border detection in dermoscopy images. Computerized Medical Imaging and Graphics 2009;33(2):148-53

[2] Wighton P, Sadeghi M, Lee TK, Atkins MS. A fully automatic random walker segmentation for skin lesions in a supervised setting. In: MICCAI (1), vol. 5762 of Lecture Notes in Computer Science. Springer; 2009. p. 1108-15.

[3] Soyer HP, Argenziano G, Chimenti S, et al. Dermoscopy of pigmented skin lesions: results of a consensus meeting via the internet. Journal of the American Academy of Dermatology 2003;48(5):679-93.

[4] Fleming MG, Steger C, Zhang J, Gao J, Cognetta AB, Pollak L, Dyer CR. Techniques for a structural analysis of dermatoscopic imagery. Computerized Medical Imaging and Graphics 1998;22(5):375-89.

[5] Fischer S, Schmid P, Guillod J. Analysis of skin lesions with pigmented networks. In: ICIP. 1996. p. 323-6.

[6] Serrano C, Acha B. Pattern analysis of dermoscopic images based on markov random fields. Pattern Recognition 2009;42(6):1052-7.

[7] Anantha M, Moss RH, Stoecker WV. Detection of pigment network in dermatoscopy images using texture analysis. Computerized Medical Imaging and Graphics 2004;28(5):225-34.

[8] Betta G, Di Leo G, Fabbrocini G, Paolillo A, Sommella P. Dermoscopic imageanalysis system: estimation of atypical pigment network and atypical vascular pattern. In: Proceedings of the IEEE International Workshop on Medical Measurement and Applications. 2006. p. 63-7.

[9] Pratt WK. Digital Image Processing. 2nd ed. New York: Wiley; 1991.

[10] Grana C, Cucchiara R, Pellacani G, Seidenari S. Line detection and texture characterization of network patterns. In: ICPR'06: Proceedings of the 18 th Inter- national Conference on Pattern Recognition. Washington, DC: IEEE Computer Society; 2006. p. 275-8.

[11] J. Kirk, Count loops in a network; 2007 [online] accessed on May 12, 2009, http://www.mathworks.com/matlabcentral/fx_files/10722/1/content/html/ run_loops_html.html.

[12] Argenziano G, Soyer HP, Giorgio VD, Piccolo D, Carli P, Delfino M, Ferrari A, Hofmann-Wellenhof R, Massi D, Mazzocchetti G, Scalvenzi M, Wolf IH. Interactive Atlas of Dermoscopy (Book and CD-ROM). Edra Medical Publishing and New Media; 2000.

Maryam Sadeghi received her BSc in Computer Engineering from Iran University of Science and Technology in 2006. She is currently pursuing a PhD in Computing Science at Simon Fraser University in the Medical Image Analysis lab. Her research focuses on computer vision and analysis of dermoscopic images.

Majid Razmara received his BSc in Computer Engineering from Iran University of Science and Technology in 2005 and the MSc in Computer Science from Concordia University in 2008. He is currently pursuing his PhD in Computing Science at Simon Fraser University on Machine Learning and Machine Translation.

Tim K. Lee received his PhD in computer science in 2001. He is currently a senior scientist at the Cancer Control Research Program, BC Cancer Agency; an assistant professor in the Department of Dermatology and Skin Science, University of British Columbia; and an adjunct professor in the School of Computing Science, Simon Fraser University. His research interests include computer-aided diagnosis and prevention of skin cancer.

M. Stella Atkins received the BSc degree in Chemistry from Nottingham University in 1966 and the $\mathrm{PhD}$ degree in computer science from the University of British Columbia in 1985. She is a Professor in the School of Computing Science at Simon Fraser University (SFU) in Vancouver, Canada, and Director of the Medical Computing Lab at SFU. Her research interests include medical image display and analysis, particularly for skin imaging, image denoising and perception, radiology workstation design and telehealth applications. She is also interested in the use of eyetrackers for improving surgery training. 